\title{
IDENTIFYING SILENT FUNGAL INFECTIONS COMPLICATING OBSTRUCTIVE UROPATHY DUE TO RENAL PAPILLARY NECROSIS IN DIABETIC PATIENTS -A STEP TOWARDS PREVENTING RECURRENT OBSTRUCTIVE UROPATHY
}

\author{
Kalyanaram Kone, Parthiban Nagaraj, Naveen Thimiri Mallikarjun, Joseph Philipraj
}

Department of Urology, Mahatma Gandhi Medical College, Puducherry, India

\section{Author for correspondence: Name: Dr. Parthiban Nagaraj: drparthiraj01@gmail.com}

\begin{abstract}
Introduction and Objectives: Renal papillary necrosis is one of the common causes of obstructive uropathy in diabetic patients. During removal of necrosed renal papilla, many patients were observed to have fluffy necrotic material in the ureter, and renal pelvis, and a few among them present again with recurrent episodes of obstructive uropathy and sepsis following DJ stent removal. Our study aimed to identify the significance of this fluffy necrotic material and its evaluation by histopathological examination (HPE).

Materials and Methods: This is a prospective observational study done in our institute by compiling data of 56 patients admitted with obstructive uropathy secondary to renal papillary necrosis who underwent a protocol-based treatment from 2016 to 2019. All these patients underwent initial DJ stenting followed by check flexible ureteroscopy or nephroscopy after 6 weeks. The white, necrotic fluffy material collected during initial DJ stenting or with subsequent flexible ureteronephroscopy was sent for HPE. All these patients were followed up for 1-3 years.

Results: Out of 56 patients, 15 patients had fluffy necrotic material in the bladder on initial cystoscopy, of which 1 patient was diagnosed with aspergillosis and 1 patient with candida infection on HPE. During check flexible ureteroscopy (FU), 19 patients had minimal burden of fluffy necrotic material in renal pelvis, of which one patient was diagnosed with aspergillosis, one with candida (same patient diagnosed on cystoscopy), and one patient with both aspergillus and candida colonies on HPE. 5 patients had the-significant burden of fluffy necrotic material in the renal pelvis, requiring removal via percutaneous nephroscopic access. Among these 5 patients, 2 were diagnosed with aspergillosis and 1 with candida infection on HPE. A total of 32 patients had single papilla, and 24 had multiple papillae in the pelvicalyceal system. 5 out of 7 patients with positive fungal pathology had negative fungal cultures. Compared to our historical data of $4 \%$ mortality and $22 \%$ recurrent obstructive uropathy in the 3 years preceding the adoption of this protocol, with the present protocol, no patient developed recurrent pyelonephritis during follow-up of 1-3 years after DJ stent removal following complete evacuation of necrotic material and appropriate antifungal treatment. Conclusion: This study highlights the need for check ureterorenoscopy and removal of all necrotic papillae and debris to establish a microbiological and histopathological diagnosis along with proper antifungal treatment to prevent episodes of recurrent pyelonephritis and obstructive uropathy.
\end{abstract}

Key-words: Renal Aspergillosis, Renal Candidiasis, Obstructive Uropathy, Papillary Necrosis, Fungal nephropathy 


\section{INTRODUCTION}

Diabetes and urologic diseases are very common health problems that markedly increase in prevalence and incidence with advancing age. Patients with type 2 diabetes mellitus are at increased risk of infections, with the urinary tract being the most frequent infection site. ${ }^{1-4}$ The spectrum of UTI in these patients ranges from asymptomatic bacteriuria (ASB) to lower UTI (cystitis), pyelonephritis, and severe urosepsis. Severe complications of UTI, such as emphysematous cystitis and pyelonephritis, renal abscesses, and renal papillary necrosis, are all encountered more frequently in type 2 diabetes than in the general population. ${ }^{5,6}$

Renal papillary necrosis can lead to secondary infection of desquamated necrotic foci, deposition of calculi, and separation and eventual sloughing of papillae, with impending acute urinary tract obstruction. Multiple sloughed papillae can obstruct their respective calyces or can congregate in the renal pelvis. Invasive fungal infections of the genitourinary tract are also increasingly recognized due to immunosuppressed status of these patients. Renal mycosis may present with varied kidney manifestations, from asymptomatic involvement to acute kidney injury. ${ }^{7,8}$ Fungal infections can co-exist with renal papillary necrosis and may lead to progressive renal inflammation.

In many patients, during initial cystoscopy and follow-up check Ureteroscopy, we have observed fluffy necrotic material in the ureter and renal pelvis. During the initial period, we did check ureteroscopy with a rigid ureteroscope and the necrotic debris was not given enough importance in the hope that it may clear with a period of DJ stenting through the dilated ureter. However, some of these patients presented with recurrent episodes of obstructive uropathy and sepsis following DJ stent removal. Initially, we had $4 \%$ mortality and $22 \%$ recurrent obstructive uropathy in the 3 years preceding the adoption of the current protocol. Following that, we changed our protocol over the last 4 years and used a flexible ureteroscope and percutaneous nephroscopy for complete inspection of pelvicalyceal system for removal of necrotic papillae and necrotic material. Our study aimed to identify the significance of this fluffy necrotic material by its evaluation with a histopathological examination
(HPE) and to see whether it can uncover any silent fungal infections. The second aim was to see whether effective antifungal treatment can reduce recurrent obstructive uropathy.

\section{MATERIALS AND METHODS}

This is a prospective observational study done in our institute after obtaining ethical committee clearance. All diabetic patients presenting with renal papillary necrosis were included in the study. The data of these patients admitted with obstructive uropathy secondary to Renal Papillary Necrosis requiring urological intervention was compiled over 4 years from 2016 to 2019. Patients with acute pyelonephritis secondary to calculi, patients with emphysematous pyelonephritis and patients who were treated elsewhere with 6 monthly or yearly DJ stent changes for obstructive uropathy were excluded from our study.

History, physical examination and radiological tests (Ultrasound and NCCT) were used to establish the diagnosis. All of these patients were started on third generation cephalosporin (Cefoperazone) after sending urine for bacterial and fungal cultures. The antibiotic was changed based on the urine culture report subsequently. After hemodynamic stabilisation, DJ stenting was done under cystoscopy guidance. Bedside PCN was done if the patient was in poor general condition and had sepsis. If the patient was stable without sepsis and if there was no purulent efflux from the ureteric orifice after passing guidewire, immediate ureteroscopy was done and necrotic papilla removed. If white fluffy necrotic material was noticed in the bladder or ureter during cystoscopy, it was retrieved and sent for histopathological analysis. Necrotic tissue was sent for histopathological analysis in formalin, and slides were processed using periodic acid Schiff(PAS) stains and reported within 72-96 hours.

Based on the fungal culture and HPE report, antifungal treatment was initiated if required. Urine bacterial and cultures were repeated after 4-6 weeks and were treated accordingly before check FU. Repeat computed tomography was done before proceeding with check ureterorenoscopy. If there is kidney bulkiness and perinephric fat stranding, check ureteroscopy was delayed for 1 month. Flexible ureterorenoscopy (FU) was done in these patients after 6 weeks, and

J Endolum Endourol Vol 4(2):e36-e44; 24 September 2021.

This article is distributed under the terms of the Creative Commons AttributionNon Commercial 4.0 International License. (c) Parthiban Nagaraj, et al. 
if patients had necrotic debris or flakes in the renal pelvis, it was evacuated either by collecting the effluent from the access sheath or by basketing. In patients with a substantial burden in the renal pelvis, percutaneous removal was done in the supine position. Those patients who had significant fungal deposits confirmed by HPE were treated with intrapelvic instillation of Amphotericin B $(50 \mathrm{mg} /$ solution at the rate of $40 \mathrm{ml} /$ hour for 5 days) through percutaneous nephrostomy along with oral Voriconazole $200 \mathrm{mg}$ BD (in aspergillosis) or Fluconazole $200 \mathrm{mg}$ OD (in candidiasis) for 1 month. Percutaneous nephrostomy was removed after 5 days of Amphotericin therapy in these patients. DJ Stent removal was done in these patients after 4 weeks. Urine cultures were repeated at 3 and 6 months for any evidence of fungal infection. All patients were followed up for a period of $1-3$ years. Statistical analysis was carried out using SPSS version 19.0 (IBM SPSS, US) software. Descriptive analyses were reported as percentage and ratio.

\section{RESULTS}

A total of 56 patients who underwent DJ stenting for 65 renal units were analysed. Among these, 35 patients were male, and 21 patients were female. 9 patients - 4 males and 5 females had bilateral obstructive uropathy. The male to female ratio was 1.7:1. The demographic characteristics are represented in Table 1. The data of patients positive for fungal elements is represented in Table 2. The flow chart depicting the treatment algorithm is represented in Figure 1.

After hemodynamic stabilisation, DJ stenting was done under cystoscopy guidance. 2 patients ( 3 renal units -1 patient bilateral) underwent bedside PCN because of poor general condition and sepsis. 22 patients (22 renal units) had immediate ureteroscopy to remove the necrotic papilla. 32 patients (40 renal units) underwent only DJ stenting. During initial cystoscopy, white fluffy material was noticed in the bladder in 15 patients, which was retrieved and sent for histopathological analysis. Among these 15 patients, histopathological examination revealed aspergillosis in 1 patient and candidiasis in another. Antifungal treatment with oral voriconazole was initiated $(200 \mathrm{mg}$ BD for 1 month) in aspergillosis and oral fluconazole (200 mg BD for 14 days) in the case of candidiasis.
Table 1. Patient characteristics

\begin{tabular}{|c|c|}
\hline Age & $38-79$ years \\
\hline Sex & $\begin{array}{l}\text { Male } 35 \text { (Bilateral 4) } \\
\text { Females } 21 \text { (Bilateral 5) } \\
\text { Ratio is 1.7:1 }\end{array}$ \\
\hline Co- Morbidity & $\begin{array}{l}\text { 56/56 Diabetes }(100 \%) \\
\text { 43/56 Hypertension( }(76.8 \%) \\
\text { 27/56 CAD }(48.2 \%) \\
\text { 17/56 chronic analgesic } \\
\text { intake }(30.35 \%) \\
0 / 56 \text { Sickle cell disease }\end{array}$ \\
\hline Involved Side & $\begin{array}{l}\text { Right } 21 / 56(37.5 \%) \\
\text { Left } 26 / 56(48.21 \%) \\
\text { Bilateral } 9 / 56(16.07 \%)\end{array}$ \\
\hline Admission & $\begin{array}{l}\text { Urology OP - } 23 \\
\text { Casualty - } 25 \\
\text { Medicine ICU - } 8\end{array}$ \\
\hline $\begin{array}{l}\text { Pre-Operative Urine } \\
\text { culture }\end{array}$ & $\begin{array}{l}\text { Sterile - } 12 \\
\text { E. coli - } 24 \\
\text { Proteus mirabilis - } 3 \\
\text { Klebsiella pneumoniae - } 3 \\
\text { Enterococcus - } 4 \\
\text { Mixed - } 3 \\
\text { Others - } 3 \\
\text { Inconclusive - } 4 \\
\text { Candida - } 12\end{array}$ \\
\hline $\begin{array}{c}\text { Pre-Check ureteroscopy } \\
\text { culture }\end{array}$ & $\begin{array}{l}\text { Sterile - } 29 \\
\text { E. coli - } 15 \\
\text { Proteus mirabilis - } 1 \\
\text { Klebsiella pneumoniae - } 2 \\
\text { Enterococcus - } 1 \\
\text { Mixed - } 3 \\
\text { Others - } 2 \\
\text { Inconclusive - } 3 \\
\text { Candida - } 6 \\
\text { Aspergillus-1 }\end{array}$ \\
\hline $\begin{array}{l}\text { Serum creatinine } \\
\qquad \begin{array}{c}<\mathrm{mg} \% \\
2-3 \mathrm{mg} \% \\
3-5 \mathrm{mg} \% \\
>5 \mathrm{mg} \%\end{array}\end{array}$ & $\begin{array}{l}27 / 56(48.21 \%) \\
18 / 56(32.14 \%) \\
6 / 56(10.71 \%) \\
5 / 56(8.92 \%)\end{array}$ \\
\hline
\end{tabular}

Initial fungal culture was negative in the patient with aspergillosis. Overall, 12 of 56 patients had positive initial fungal culture.

After 6 weeks, repeat computed tomography and urine cultures were done before checking ureterorenoscopy and DJ stent removal. 7 patients had positive fungal culture and were treated before FU. 
Table 2. Data of patients positive for fungal elements

\begin{tabular}{|c|c|c|c|c|c|c|c|}
\hline S.no & Age & Sex & $\begin{array}{c}\text { Preop } \\
\text { Bacterial culture }\end{array}$ & $\begin{array}{c}\text { Preop } \\
\text { Fungal culture }\end{array}$ & Procedure & $\begin{array}{l}\text { Post op } \\
\text { HPE }\end{array}$ & REMARKS \\
\hline 1 & 44 yrs & $\mathrm{F}$ & E. coli & Candida & $\begin{array}{l}\text { Cystoscopy } \\
\text { and Flexible } \\
\text { URS }\end{array}$ & Candida & $\begin{array}{l}\text { Received } 2 \text { courses - } \\
\text { initially advised for } 2 \\
\text { weeks (Patient took only } \\
\text { for } 5 \text { days and stopped) } \\
\text { and then } 1 \text { month } \\
\text { Fluconazole treatment } \\
\text { (Started } 1 \text { week before } \\
\text { Check URS) }\end{array}$ \\
\hline 2 & $59 y r s$ & $\mathrm{M}$ & Sterile & Sterile & Cystoscopy & Aspergillosis & Voriconazole for 1 month \\
\hline 3 & $67 \mathrm{yrs}$ & M & Sterile & Sterile & Flexible URS & Aspergillosis & $\begin{array}{l}\text { Bilateral Flexible URS } \\
\text { Voriconazole for } 1 \text { month }\end{array}$ \\
\hline 4 & $72 \mathrm{yrs}$ & M & E. coli & Sterile & Flexible URS & $\begin{array}{l}\text { Candida \& } \\
\text { Aspergillosis }\end{array}$ & $\begin{array}{l}\text { After biopsy report - } \\
\text { Voriconazole for } 1 \text { month }\end{array}$ \\
\hline 5 & $60 \mathrm{yrs}$ & M & E. coli & Sterile & $\begin{array}{c}\text { Bilateral } \\
\text { percutaneous } \\
\text { nephroscopy }\end{array}$ & Aspergillosis & $\begin{array}{l}\text { Pre-URS Fluconazole for } \\
1 \text { week and for } 5 \text { days } \\
\text { after URS Post-op. } \\
\text { Amphotericin for } 5 \text { days } \\
\text { through PCN } \\
\text { Voriconazole for } 1 \text { month }\end{array}$ \\
\hline 6 & $60 \mathrm{yrs}$ & M & Klebsiella & Candida & $\begin{array}{l}\text { Percutaneous } \\
\text { nephroscopy }\end{array}$ & Candida & $\begin{array}{l}\text { Post-op Amphotericin for } \\
5 \text { days through PCN } \\
\text { Fluconazole for } 1 \text { month }\end{array}$ \\
\hline 7 & $55 \mathrm{yrs}$ & M & Enterococcus & Sterile & $\begin{array}{l}\text { Percutaneous } \\
\text { nephroscopy }\end{array}$ & Aspergillosis & $\begin{array}{l}\text { Post-op Amphotericin for } \\
5 \text { days through PCN } \\
\text { Voriconazole for } 1 \text { month }\end{array}$ \\
\hline
\end{tabular}

Repeat non-contrast computed tomography (NCCT) revealed a reduction in the kidney bulkiness and perinephric fat stranding in all except 5 patients. In these 5 patients, check ureteroscopy was delayed for 1 month. In 32 patients ( 34 renal units - 2 patients had bilateral obstructive uropathy), 20 patients had no papilla, and 12 had single papilla in ureter with the clear renal pelvis on check ureterorenoscopy. In 19 patients ( 24 renal units -5 patients had bilateral obstructive uropathy), check URS revealed fluffy necrotic tissue in the renal pelvis. Out of 5 patients who had bilateral obstructive uropathy, only 1 patient had bilateral deposits on endoscopy. Flexible ureterorenoscopy (RIRS) was done to remove the fluffy material in these patients. Additional papillae lying in other calyces were removed in these patients. Specimen sent for histopathological analysis revealed aspergillosis in one patient, candida infection in one patient, and one patient had mixed colonies of both aspergillosis and candida. The patient with only candida isolate was the same patient who had candida colonies on initial cystoscopy. This patient defaulted on treatment during the initial episode and was treated again with oral fluconazole for 1 more month. All these patients had negative fungal cultures before the procedure.

In 5 patients ( 7 renal units -2 patients had bilateral obstructive uropathy), flexible ureterorenoscopy showed a huge burden of fluffy necrotic tissue in the renal pelvis, which was not amenable for safe and quick ureteroscopic retrieval. So, we proceeded with a percutaneous renal puncture in the supine position for its retrieval via mini nephroscopy. In one patient, we did simultaneous bilateral percutaneous retrieval. One patient had fluffy necrotic deposits occupying the entire renal pelvis and calyces. Entire fluffy necrotic tissue was removed in all these patients, and percutaneous nephrostomy was placed. Histopathological analysis reports revealed aspergillosis in two patients 


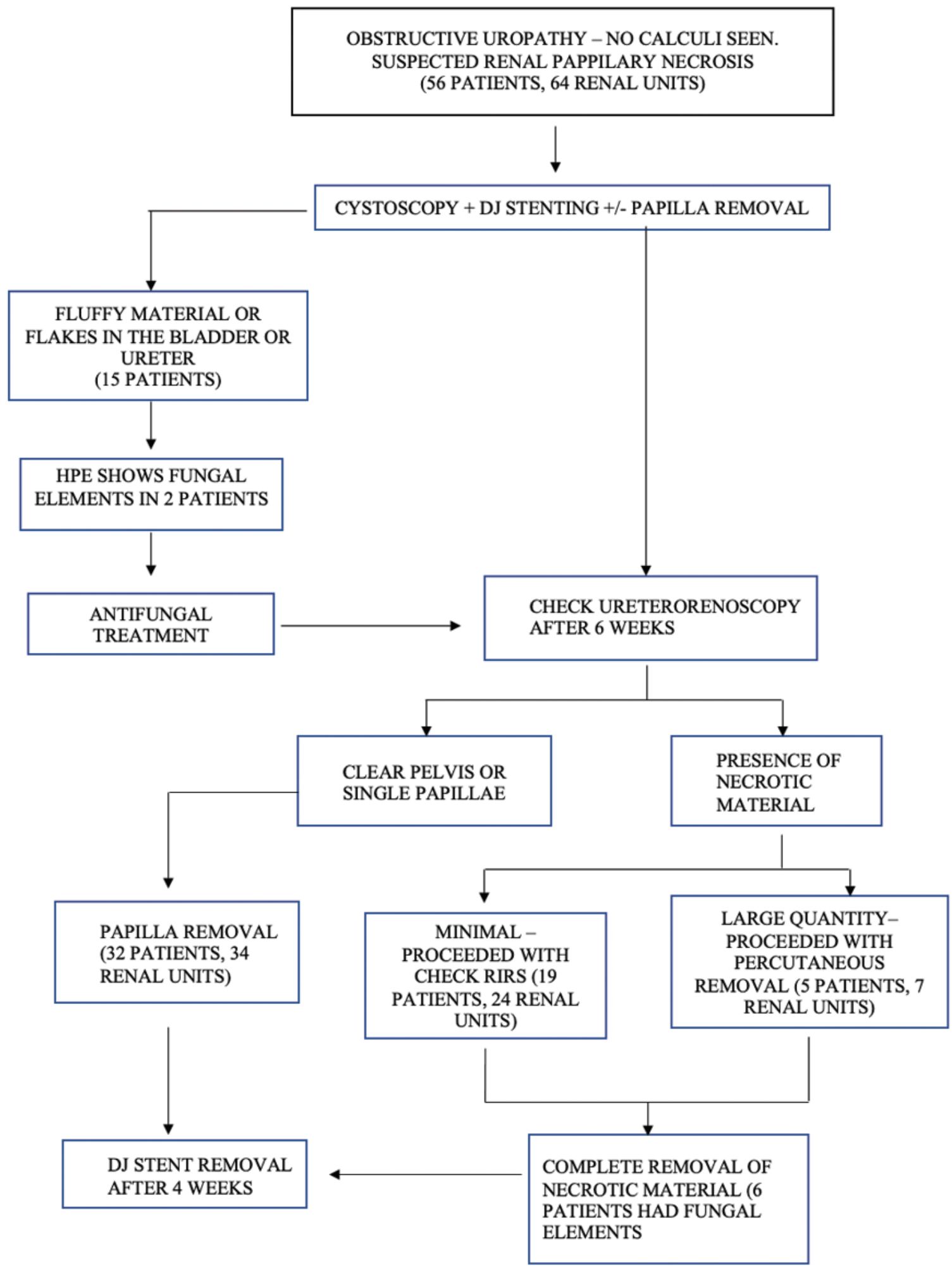

Figure 1. Treatment algorithm. Non Commercial 4.0 International License. (c) Parthiban Nagaraj, et al. 
and candida infection in one patient. Two patients had debris, a mixture of necrotic papillae with proteinaceous deposits without any fungal deposits on histopathology. The three patients who had fungal deposits were treated with intrapelvic instillation of Amphotericin B $(50 \mathrm{mg} / \mathrm{solution}$ at the rate of $40 \mathrm{~mL} /$ hour for 5 days) through percutaneous nephrostomy along with oral voriconazole $200 \mathrm{mg}$ BD (in aspergillosis) or Fluconazole $200 \mathrm{mg}$ OD (in candidiasis) for 1 month. Percutaneous nephrostomy was removed after 5 days of amphotericin therapy in these patients. DJ Stent removal was done in these patients after 4 weeks. Out of these 3 patients, only one patient with candidiasis had positive fungal culture.

All the patients had uneventful recovery following check ureteroscopy or nephroscopy. 11 patients had postoperative fever, requiring a change of antibiotics (Clavien-Dindo Class 2). None of the patients developed sepsis. Urine for fungal culture was sent for all patients at 6 weeks of follow-up, which did not grow any fungal elements in any of the treated patients. We had follow-up of 45 out of 56 patients and all the 7 patients who had renal fungal involvement on HPE. In patients with invasive aspergillosis, repeat check URS was done after 6 months, which did not show any necrotic tissue. None of these patients presented with a recurrent episode of pyelonephritis or obstructive uropathy during the 1-3 years follow-up.

\section{DISCUSSION}

Renal papillary necrosis (RPN) is characterised by ischaemic coagulative necrosis of the renal papilla which is triggered by multiple associated conditions. The distinctively less blood supply of the renal medulla, especially at the tip of the papilla, and its hypertonic environment make the area prone to vascular compromise. Various causes for ischaemia are proposed - arteriosclerosis, vascular spasm, medullary vasculature embolism or thrombosis, compression of vessels by inflammation or edema or increased intrapelvic pressure, and accumulation of small abscesses at the base of papilla., ${ }^{9,10}$

RPN signifies a field change disease in the affected kidney, and in people with diabetes, it is the end result of atherosclerosis, infection, and obstruction in varying proportions. After relieving the obstruction, if the infection is not eliminated entirely, it may lead to a vicious cycle of slow destruction of the remaining renal papillae and further obstructive cycles in some patients. The necrotic tissue also serves as a nidus for secondary bacterial or fungal infection and deposition of calculi. Even in classic bacterial pyelonephritis with repeated positive urine cultures for bacteria, refractory to antimicrobials, a fungal infection might co-exist. ${ }^{11}$

The first aim of our study was to find out the nature of the necrotic material. In our study, out of 56 patients, 24 patients had necrotic material in the Urinary Tract. 7 (9 renal units) of these patients had necrotic tissue positive for fungal elements on histopathology ( $29 \%$ in patients having necrotic tissue and $14 \%$ overall). Candida infection was found in 2 patients, aspergillosis was found in 4 patients while 1 patient had mixed colonies of Candida and Aspergillosis. 5 out of these 7 patients had negative fungal cultures before the procedure. 2 patients had fungal growth found at the time of stenting (1 Candidiasis and 1 Aspergillosis). Five patients had fungal growth found at the time of check endoscopy 1-2 months after stenting. The growth of Candida species was expected in this group of patients but finding aspergillosis is entirely unexpected. Indwelling urinary drainage devices is one of the risk factors for fungal colonisation or invasive fungal infection. However, among our patients, only 1 patient who had mixed growth had an indwelling catheter.

Urine fungal cultures and radiological studies were not always helpful in identifying the fungal involvement in the disease process. Only 2 out of 7 patients had positive fungal cultures, especially in patients with candidiasis. No patient who had renal aspergillosis had positive fungal cultures. One of the cultures was contaminated, one was reported as fungal hyphae and was initially treated as candidiasis, and the other two had negative fungal cultures. Histopathological analysis of the retrieved necrotic tissue helped establish the diagnosis in all these patients (Figures 2,3,4). CECT helps find hypodense necrotic material as filling defects in the pyelogram phase. Nevertheless, many of our patients had borderline renal function, so contrast studies could not be done. Non-contrast CT scans were unable to find out the necrotic debris involving the pelvicalyceal system except in the patient with bilateral renal involvement for whom we did bilateral mini percutaneous 
removal. Even in that patient, the bulk of the material was in the right kidney, which was not recognised on NCCT (Figure 5). These findings underscore the importance of doing check ureterorenorenoscopy to find the presence of debris and remove it before DJ stent removal.

Every fungal infection has a specific characteristic mode of tissue injury and depends on which structure they invade. Candida is the most common pathogen, followed by Aspergillus. Candida normally inhabits the skin, genital and gastrointestinal tract. Finding candida in the urine is rare in patients without any risk factors. Diabetes, antibiotics usage, urinary drainage devices are the significant risk factors for Candiduria. There are three transmission routes: ascending infections (usually from indwelling bladder catheters), trauma or surgical interventions, and haematogenous spread (common in immunocompromised patients). ${ }^{12-14}$ The unique ability of candida to colonise as well as invade the urinary tract makes it difficult to determine whether the fungal growth found in patients is asymptomatic candiduria or invasive candidemia. However, the clinical setting in which the infection is found makes it mandatory to treat it as an invasive infection. For patients who have persistent candiduria and whose symptoms suggest pyelonephritis, fluconazole is the treatment of choice. The recommended dosage is 200 to $400 \mathrm{mg}$ daily for 2 weeks. For fungal balls, local instillation is only an interim procedure until surgical or interventional radiological techniques remove it. Nephrectomy is recommended for patients who have an extensive parenchymal fungal infection, particularly those who fail to respond to initial medical and endourological management. ${ }^{13,15}$

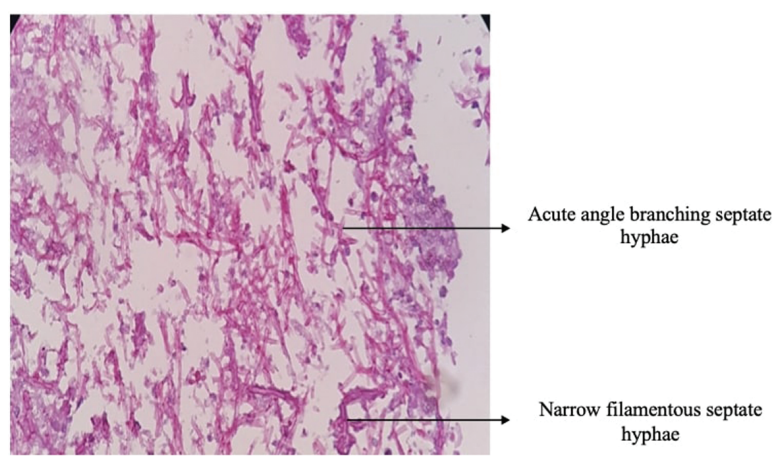

Figure 2. Microscopic view depicting clusters of narrow filamentous acute angle branching septate hyphae suggestive of aspergillosis.
Aspergillus species are filamentous, environmental fungi that cause a spectrum of infections ranging from hypersensitivity reactions to acute life-threatening infections, the latter often occurring primarily in immunocompromised individuals ${ }^{16}$. Analysis of a large series of patients with fungal infections of the kidney revealed that Aspergillus infection constituted about $30 \%$ of total renal fungal infection and the majority (63\%) occurred from disseminated infection. Primary renal aspergillosis in diabetic patients is rare. The majority of them are insidious, and their diagnosis

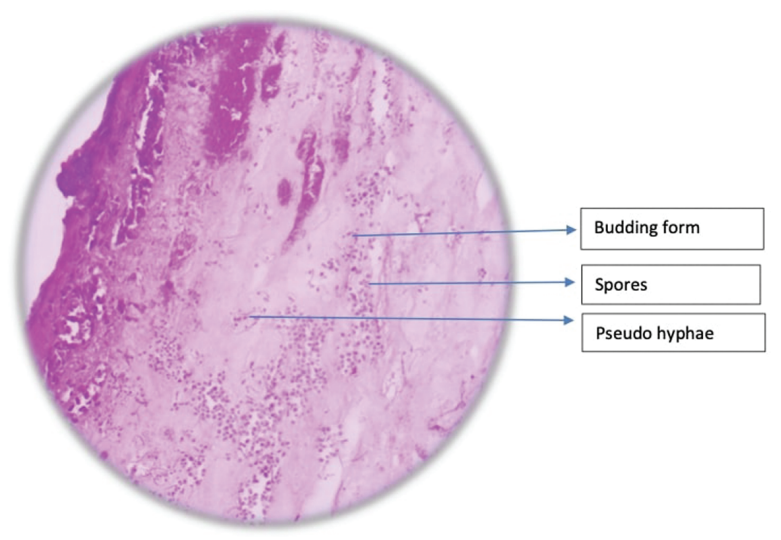

Figure 3.Microscopic view depicting numerous spores, budding and pseudo hyphae suggestive of candidiasis.

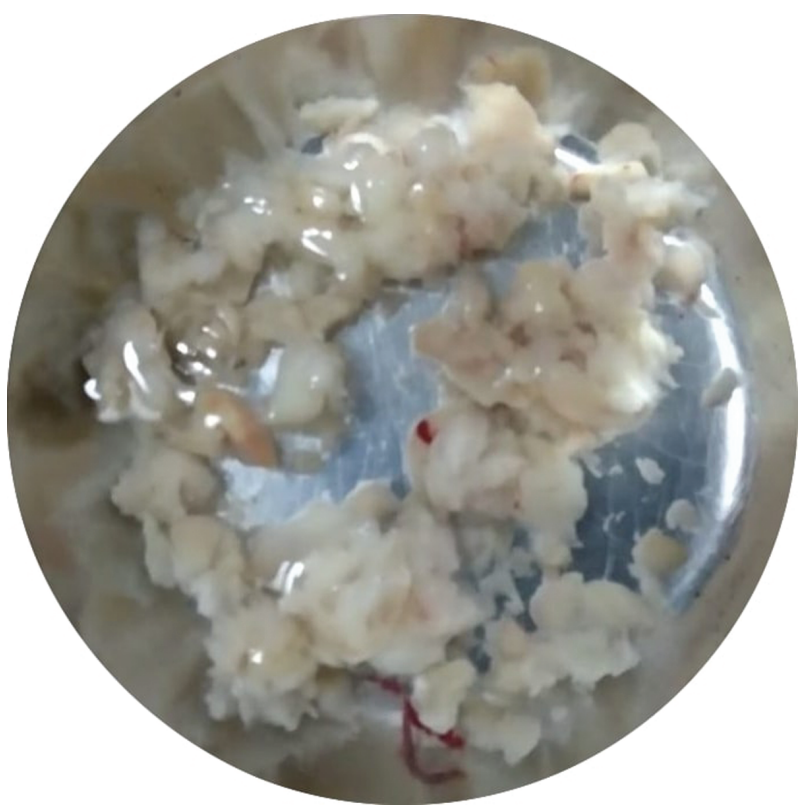

Figure 4. Depiction of sloughy necrotic debris retrieved intra-operatively. 

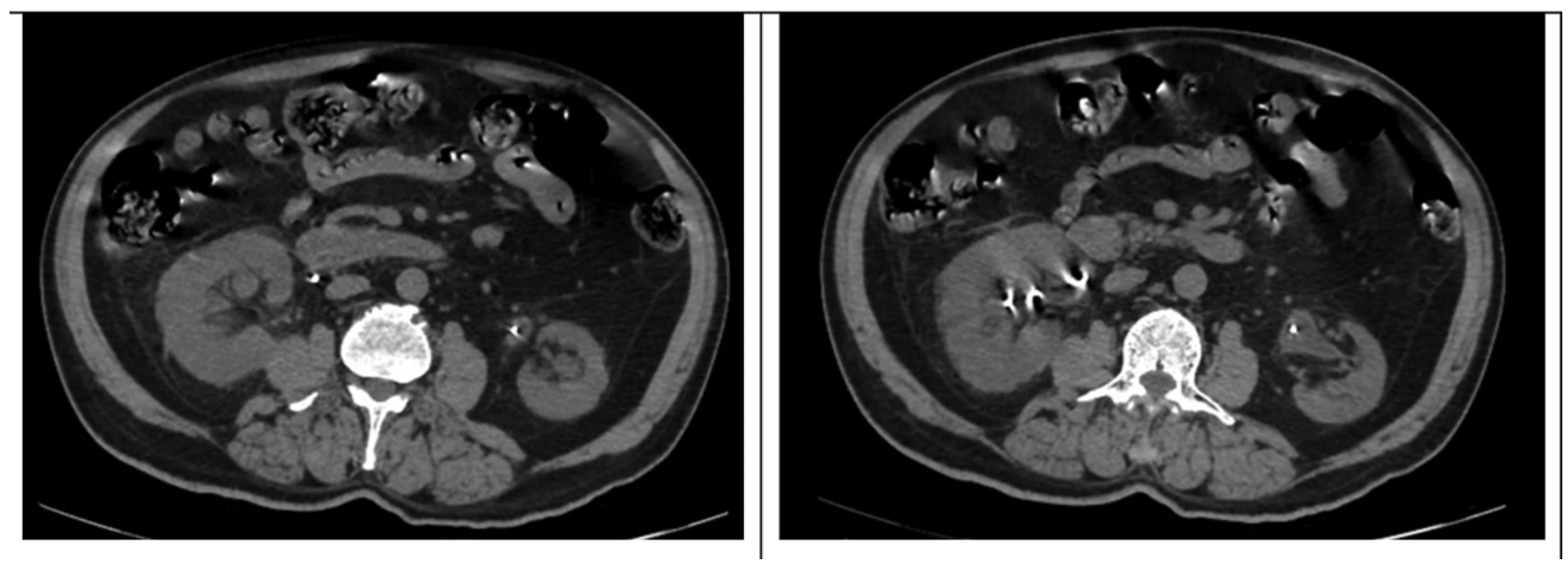

Figure 5.Pre-Check Ureterorenoscopy NCCT KUB images showing left hydroureteronephrosis with bulky right kidney. (Note: Intra-operatively bulk of the necrotic material was in the right kidney which was not $\operatorname{recogn}$ on NCCT).

is usually delayed because of other co-existing illnesses. Renal aspergillosis may present in different presentations: disseminated aspergillosis with renal involvement resulting from haematogenous spread to the kidneys leading to the formation of multi-focal abscesses; aspergillosis of the renal pelvis with bezoars formation resulting in obstructive uropathy and ascending pan-urothelial aspergillosis. Aspergillus bezoars (fungal balls) may fill the renal pelvis, causing hydronephrosis, and these masses may be passed in the urine, causing renal colic. Infections caused by Aspergillus spp. are associated with high morbidity and mortality. ${ }^{16-18}$ The excellent recovery rates found in our patients may be because of isolated renal pelvic involvement rather than disseminated infection.

Management of urinary tract aspergillosis, especially isolated renal pelvis involvement, remains a therapeutic challenge in our current clinical scenario. Current Infectious Diseases Society of America guidelines has recommended a combined medical and surgical management for renal aspergillosis. Systemic administration of oral voriconazole $200 \mathrm{mg}$ twice a day for 2 weeks to 1 month combined with topical instillation of amphotericin B deoxycholate is recommended. ${ }^{16}$ Nephrostomy tube can be used for intrapelvic instillation with amphotericin B deoxycholate to achieve high local concentrations. Given this route, amphotericin $\mathrm{B}$ is not absorbed and is not nephrotoxic. ${ }^{16}$

The second aim of our study was to find out whether the removal of the necrotic material has any effect on the postoperative course by preventing recurrent obstructive episodes and pyelonephritis. We had follow-up of 45 out of 56 patients and all the 7 patients who had renal fungal involvement. We had historical data of patients who presented with similar illnesses in the 3 years before adopting this protocol which showed a mortality rate of $4.4 \%$ (2 out of 45 patients) and recurrent obstructive uropathy of $22 \%$ (10 out of 45 patients). In comparison, none of the present protocol patients presented with recurrent episodes of pyelonephritis or obstructive uropathy during the follow-up period of 1-3 years. This supports our hypothesis that removing the necrotic material has a beneficial effect on the patient outcome.

We want to emphasise that renal fungal involvement especially aspergillosis is not infrequent in this group of patients, and steps should be taken to treat the infection early so that it may not become an invasive infection later on with its attendant morbidity.

\section{CONCLUSION}

Urine fungal cultures and radiological studies were not always helpful in identifying the Renal fungal involvement. Histopathology of evacuated necrotic material attains importance in this scenario. The nature of the necrotic material revealed that almost 30 percent of patients who had this, had fungal involvement, mainly aspergillosis. Aspergillosis of the Urinary tract is not as infrequent as thought, and a search should be done in this group of patients for

J Endolum Endourol Vol 4(2):e36-e44; 24 September 2021.

This article is distributed under the terms of the Creative Commons AttributionNon Commercial 4.0 International License. (c) Parthiban Nagaraj, et al. 
silent renal fungal involvement. Under proper coverage of antibiotics and antifungals and after a period of DJ stenting, aggressive removal of this material can be done safely without any adverse complications. After removing the fluffy necrotic tissue, no patient had recurrent pyelonephritis, which supports the hypothesis that it may improve the patient outcome. There are certainly some limitations in our study. Our study is only an observational study comparing with historic data and not a randomised study. The number of patients treated is also small, and a single small study cannot give definite recommendations. Further studies recruiting a more significant number of patients can be undertaken by multiple institutions so that solid advice can be given.

\section{ACKNOWLEDGEMENT}

Nil.

\section{GRANT SUPPORT}

Nil.

\section{CONFLICT OF INTEREST}

Nil.

\section{REFERENCES}

1. Patterson JE, Andriole VT. Bacterial urinary tract infections in diabetes. Infect Dis Clin North Am 1997;11(3):735-750.

2. Joshi N, Caputo GM, Weitekamp MR, Karchmer AW. Infections in patients with diabetes mellitus. N Engl J Med 1999;341(25):1906-1912.

3. Boyko EJ, Fihn SD, Scholes D, Abraham L, Monsey B. Risk of urinary tract infection and asymptomatic bacteriuria among diabetic and nondiabetic postmenopausal women. Am J Epidemiol 2005;161(6):557-564.

4. Shah BR, Hux JE. Quantifying the risk of infectious diseases for people with diabetes. Diabetes Care 2003;26(2):510-513.

5. Kofteridis DP, Papadimitraki E, Mantadakis E, et al. Effect of diabetes mellitus on the clinical and microbiological features of hospitalized elderly patients with acute pyelonephritis. J Am Geriatr Soc 2009;57(11):2125-2128.

6. Mnif MF, Kamoun M, Kacem FH, et al. Complicated urinary tract infections associated with diabetes mellitus: pathogenesis, diagnosis and management. Indian J Endocrinol Metab 2013;17(3):442-445.

7. Wise GJ, Silver DA. Fungal infections of the genitourinary system. J Urol 1993; 149: 1377-1388

8. Gupta KL, Joshi K, Sud K et al. Renal zygomycosis: an underdiagnosed cause of acute renal failure. Nephrol Dial Transplant 1999;14: 2720-2725

9. Abdulhayoglu S., Marble A. Necrotizing renal papillitis (papillary necrosis) in diabetes mellitus. Am J Med Sci 1964 Dec;248:623-32.

10. Jung DC, Kim SH. Jung SI, et al. Renal Papillary Necrosis: Review and Comparison of Findings at Multi-Detector Row CT and Intra-venous Urography. RadioGraphics 2006 26:6, 1827-1836.

11. Mitchell KM. Co-existing bacterial pyelonephritis and bilateral ureteral fungus balls in a diabetic patient. Case report. J Fla Med Assoc 1990;77(6):596-599.

12. Fisher JF. Candida urinary tract infections - epidemiology, pathogenesis, diagnosis, and treatment: executive summary. Clin Infect Dis 2011;52:S429-32.

13. Kauffman CA. Diagnosis and management of fungal urinary tract infection. Infect Dis Clin North Am 2014 Mar;28(1):61-74.

14. Colodner R, Nuri Y, Chazan B, et al. Communityacquired and hospital-acquired candiduria: comparison of prevalence and clinical characteristics. Eur J Clin Microbiol Infect Dis 2008;27:301-5.

15. Chitale SV, Shaida N, Burtt G, et al. Endoscopic management of renal candidiasis. J Endourol 2004;18:865-6.

16. Patterson TF, Thompson GR, Denning DW, et al. Practice Guidelines for the Diagnosis and Management of Aspergillosis: 2016 Update by the Infectious Diseases Society of America. Clin Infect Dis. 2016;63:e1-e60

17. Gupta KL: Fungal infections and the kidney. Indian J Nephrology 2001;11:147-154.

18. Jenks, J.D.; Hoenigl, M. Treatment of Aspergillosis. J Fungi 2018;4:98. 